\title{
Conflictos socioambientales y complejidad
}

\author{
Dr. Rodrigo Arce \\ Universidad Ricardo Palma (Perú) Universidad \\ Nacional de Ingeniería UNI (Perú) \\ Universidad Nacional Mayor de San Marcos (Perú) \\ rarcerojas@yahoo.es
}

Recepción: 20/05/2021

Aceptación: 12/06/2021

\section{Propósito}

El propósito de este artículo es contribuir a la comprensión de las relaciones entre los conflictos y la complejidad desde la perspectiva de las ciencias de la complejidad. Para el efecto se realizó una investigación bibliográfica crítica que se suma a la experiencia del autor en procesos de diálogo y transformación de conflictos en contextos de industrias extractivas. El artículo tiene carácter reflexivo y propositivo. El ámbito de investigación corresponde al Perú pero tiene alcances Latinoamericanos en tanto se comparte mayoritariamente el modelo económico neoliberal.

\section{Hallazgos}

Se indican los siguientes hallazgos:

- La comprensión de las relaciones entre los conflictos socioambientales y la complejidad, desde la perspectiva de las ciencias de la complejidad, ha permitido poner en evidencia el enriquecimiento del abordaje de los conflictos al ampliar significativamente los elementos, relaciones y procesos en sistemas abiertos que intercambian materiales y energía con el entorno.

- Entender los conflictos socioambientales en perspectiva de complejidad implica incorporar el respeto por los valores intrínsecos de la vida en todas sus manifestaciones que había sido negada o subestimada por la primacía de consideraciones de crecimiento económico y una estructura de gestión pública sectorial y disciplinaria que no da cuenta de la complejidad de la realidad.

\section{Implicaciones sociales}

El artículo contribuya a revisar los enfoques convencionales de gestión de conflictos que generalmente buscan desconflictuar para que las actividades económicas que impactan al ambiente y la sociedad puedan seguir operando gracias a acuerdos compensatorios o transaccionales. El enfoque invita a pensar el manejo de conflictos socioambientales desde una perspectiva más integral que apunta a una mejor gobernanza. Otra gran implicancia es la revisión de las relaciones con la naturaleza de la cual formamos parte pero que hasta ahora se ha invisibilizado o subestimado al reducir a los ecosistemas como fuentes de materias primas o capital natural.

\section{Originalidad / valor}

La relación entre los conflictos socioambientales y la complejidad aún es débilmente 
abordada en el medio tanto por la reciente valoración de las ciencias de la complejidad como por el hecho que el modelo estándar de manejo de conflictos está fuertemente marcado por el control y la urgencia de atender las crisis.

\section{Declaración de conflicto de intereses}

El autor declara que no tiene conflicto de intereses que hayan sesgado la elaboración del artículo.

\section{Resumen}

El presente artículo trata de la comprensión de los conflictos socioambientales desde la perspectiva de la complejidad con base en una revisión bibliográfica crítica y la experiencia del autor en procesos de diálogo y conflictos en contextos de industrias extractivas. Se concluye que la comprensión de las relaciones entre los conflictos socioambientales y la complejidad, desde la perspectiva de las ciencias de la complejidad, ha permitido poner en evidencia el enriquecimiento del abordaje de los conflictos al ampliar significativamente los elementos, relaciones y procesos en sistemas abiertos que intercambian materiales y energía con el entorno. Entender los conflictos socioambientales en perspectiva de complejidad implica incorporar el respeto por los valores intrínsecos de la vida en todas sus manifestaciones que había sido negada o subestimada por la primacía de consideraciones de crecimiento económico y una estructura de gestión pública sectorial y disciplinaria que no da cuenta de la complejidad de la realidad. Consecuentemente surge la necesidad de revisar el modelo capitalista neoliberal hegemónico que imprime una lógica y sentido al manejo de conflictos para desconflictuar. Es entonces que cobra sentido la propuesta de biodesarrollo que está acompañada de una ética ambiental y ecológica.

Palabras clave: Ciencias de la complejidad, cultura, naturaleza, ontología, poder.
Abstract
This article deals with the understanding of socio-envi- ronmental conflicts from the perspective of complexity based on a critical literature review and the author's experience in dialogue and conflict processes in the context of extractive industries. It is concluded that the understanding of the relationships between so- cio-environmental conflicts and complexity, from the perspective of complexity sciences, has made it possible to highlight the enrichment of the approach to conflicts by significantly broadening the elements, relationships and processes in open systems that exchange mate-

rials and energy with the environment. Understanding socio-environmental conflicts from a complexity perspective implies incorporating respect for the intrinsic values of life in all its manifestations, which had been denied or underestimated by the primacy of economic growth considerations and a sectoral and disciplinary public management structure that does not take into account the complexity of reality. Consequently, there is a need to review the hegemonic neoliberal capitalist model that imprints a logic and meaning on conflict management in order to deconflict. It is then that the proposal of biodevelopment, which is accompanied by an environmental and ecological ethics, makes sense.

Key words: Complexity sciences, culture, nature, ontology, power.

\section{Introducción}

El presente artículo trata de la comprensión de los conflictos socioambientales desde la perspectiva de la complejidad desde el contexto peruano aunque las reflexiones pueden proyectarse a los países Latinoamericanos que comparten el sistema económico neoliberal. Aunque mayoritariamente se reconoce la "complejidad" de los conflictos socioambientales la comprensión de lo complejo está dada más bien por su carácter complicado y no tanto desde cómo se entiende la complejidad desde las ciencias de la complejidad, proceso entendible además por la reciente irrupción de las ciencias de la complejidad en el ambiente institucional y académico peruano.

Más allá de cualquier discurso teórico, ideológico o ideologizado lo cierto es que el planeta ha superado los umbrales ecológicos en cuanto al cambio climático, pérdida de biodiversidad, cambio de uso de la tierra y alteraciones de los ciclos del fósforo y carbono fundamentalmente producto de la actividad agrícola industrial (Raworth, 2018). La Comisión Económica para América Latina y el Caribe (CEPAL, 2021, p. 83) refiere que "la pérdida de bosques tropicales y subtropicales tiene un fuerte impacto en la biodiversidad y la hidrología de las que dependen los sistemas económicos, por lo que su protección es vital." Como señala WWF (2020, p.2) "La destrucción de la naturaleza por parte de la humanidad está teniendo impactos catastróficos, no solo en las poblaciones de fauna y flora salvajes, sino también en la salud humana y en todos los aspectos de nuestras vidas." Los conflictos socioambientales por tanto involucran a toda la realidad (o realidades) imbricada (s) y es necesario su entendimiento para el despliegue de visiones y acciones transformativas (Arce 2016, 2018). 
Existen diferentes escuelas o corrientes relativos a los conflictos, así se habla de tratamiento, resolución, manejo, gestión y transformación de conflictos en una suerte de reconocimiento de la complejidad creciente de los conflictos. La primera orientación (tratamiento, resolución, manejo y gestión) presenta enfoques más orientados al control del conflicto mientras que la segunda orientación (la transformación de conflictos) tiene un enfoque más estructural porque alude a cambios personales, relacionales, culturales y estructurales. De otro lado, las ciencias de la complejidad se ocupan de los fenómenos de complejidad creciente tales como las crisis, las singularidades, los eventos raros, entre otros. Las ciencias de la complejidad se concentran en las irrupciones, quiebres, fracturas, bifurcaciones, y otros fenómenos o atributos, desde una perspectiva de síntesis. Contrario a una ciencia normal que estandariza el rol de las ciencias de la complejidad es indeterminar, desequilibrar, otorgar grados de libertad (Maldonado, 2016a). Las ciencias de la complejidad surgen "como una nueva forma de hacer Ciencia investigando los sistemas humanos y sociales, no como sistemas mecanicistas sino como sistemas interactivos, dinámicos, no lineales, y provistos de un sentido teleológico, capaces de adaptación, aprendizaje e innovación, y con una tendencia a estructurarse en redes complejas." (Lara, 2016, p. 43). De ahí que resulte sugestivo entender los conflictos socioambientales desde la perspectiva de las ciencias de la complejidad.

El abordaje de los conflictos se realiza con un vocabulario fuertemente fisicalista así se habla de masas, acción-reacción, energía, fuerzas, poder, resistencias, tensiones, colisiones, siempre con un enfoque binario como por ejemplo individuo-sociedad, hombre-naturaleza, razón-emoción, lógico-ilógico, que resultan insuficientes para explicar la complejidad de la realidad que presenta traslapes, enredos, entreveros (Maldonado, 2016a). Los conflictos socioambientales se ubican en contextos rizomáticos que superan ampliamente los enfoques lineales (Silva, 2017).

Las relaciones entre conflictos socioambientales y complejidad aún no han sido suficientemente tratadas y es propósito del presente artículo visualizar estas relaciones con la finalidad de contribuir a abordajes más estratégicos que superen el enfoque de las ciencias normales que todavía permea en gran medida en la gestión de los conflictos socioambientales.

La pregunta que ha guiado la reflexión es ¿Cuáles son las relaciones entre los conflictos ambientales y la complejidad? Para el efecto se ha desarrollado la siguiente línea argumentativa: Ios conflictos sociales, los conflictos socioambientales, y la complejidad de los conflictos socioambientales. Se realizó las discusiones y se derivaron conclusiones.

\section{La conflictividad social}

La conflictividad social es definida como una dinámica de oposiciones entre sectores de la sociedad (poblaciones), el Estado y las Empresas que perciben que sus objetivos, intereses, necesidades, valoraciones, percepciones, significados y sentidos son contradictorios en acciones colectivas que se manifiestan como un proceso complejo en los ámbitos públicos que en ocasiones pueden derivar en violencia y amenaza a la gobernabilidad (Secretaría de Gestión Social y Diálogo del Viceministerio de Gobernanza Territorial de la Presidencia del Consejo de Ministros, 2019, Defensoría del Pueblo, 2021). Nótese que la mirada desde el Estado refiere a las amenazas a la gobernabilidad, entendida como ejercicio del poder, por lo tanto estado-céntrica.

El hecho que los conflictos tengan una dimensión intangible que incluye cosmovisiones, valores y sentidos lleva a reconocer precisamente a reconocer la importancia de la interculturalidad en el abordaje de los conflictos. Se requiere no quedarse en una perspectiva de interculturalidad funcional y se avance hacia una interculturalidad crítica que sea capaz de dar cuenta de las relaciones asimétricas de poder (Rodríguez et al., 2015).

\section{Los conflictos socioambientales}

Específicamente un conflicto socioambiental, desde la perspectiva de la Defensoría del Pueblo (2021, p. 4) están referidos "al control, uso y/o acceso al ambiente y sus recursos." La Defensoría del Pueblo aclara que en estos conflictos "Están presentes también componentes políticos, económicos, sociales y culturales." En el mismo sentido la Comissão Pastoral da Terra - CPT (2020, p. 10) habla específicamente de conflictos socioterritoriales que en la práctica son al mismo tiempo conflictos sociales y económicos en tanto alude a la interacción entre el ecosistema natural y todos aquellos seres que lo habitan y que dependen de él para sobrevivir. Silva (2017, p. 14) cuestiona la denominación de conflictos socioambientales y los denomina conflictos ecoterritoriales en tanto refieren a disputas sobre el uso y gestión de los territorios que implican afectación ambiental. En este artículo se seguirá usando el término conflictos socioambientales como categoría de trabajo pero se asume que incluye las perspectivas de los conflictos socioterritoriales y los conflictos ecoterritoriales.

El entendimiento más frecuente de los conflictos socioambientales refiere a pugnas por el acceso, uso, 
control, toma de decisiones y distribución de beneficios relativos al uso de los recursos naturales que ofrece el ambiente (Castro, 2013). Gómez (2021) añade que también refiere a la gestión y valoración de bienes comunes, territorios y ecosistemas. Es preciso señalar que los conflictos socioambientales se ubican en la esfera pública por lo que su solución requiere la presencia del Estado (Morales et al., 2019).

Por su parte Walter (2009) señala que los autores diferencian entre conflictos ambientales que se refieren a aquellos conflictos cuya oposición fundamentalmente provienen de activistas externos y los conflictos socioambientales cuya oposición involucra a las comunidades involucradas. Nótese que esta forma de entender los conflictos surge principalmente desde la mirada externa a los actores comunitarios.

Los conflictos socioambientales pueden basarse en situaciones reales o aparentes (inexactas o estereotipadas) (Carpio et al., 2006). En todo caso, la percepción juega un rol fundamental porque independientemente si corresponde o no a la realidad influye poderosamente en las acciones (Castro, 2013). Además es necesario señalar que los conflictos pueden fundamentarse en aspectos materiales o simbólicos.

Existen diversos enfoques respecto a los conflictos socioambientales que se muestran en la figura 1 y que dan cuenta de aproximaciones desde diversos planos que podrían resumirse como ontológico (relacional), valorativo (paradigmático y simbólico) y un plano material (los medios de vida) aunque todos estos planos se ubican en una categoría mayor que es el territorio, tal como lo conciben los pueblos indígenas y no desde el concepto legal de territorio que alude exclusivamente a la dimensión biofísica. Al respecto Ortiz et al. (2011) acotan que los conflictos son procesos que surgen de las relaciones existentes que reflejan las interacciones pasadas y presentes en un contexto dado. Rodríguez et al. (2015) plantean que los conflictos socioambientales aluden a conflictividades más amplias, el poder y la cultura. Por su parte Bebbington y Humphreys (2009, p. 117) identifican "cinco tipos de ambientalismos que están presentes en los conflictos mineros en el Perú: el conservacionista, el nacionalista-populista, el de defensa de las formas de vida, el de justicia socio-ambiental y el ecologismo profundo."

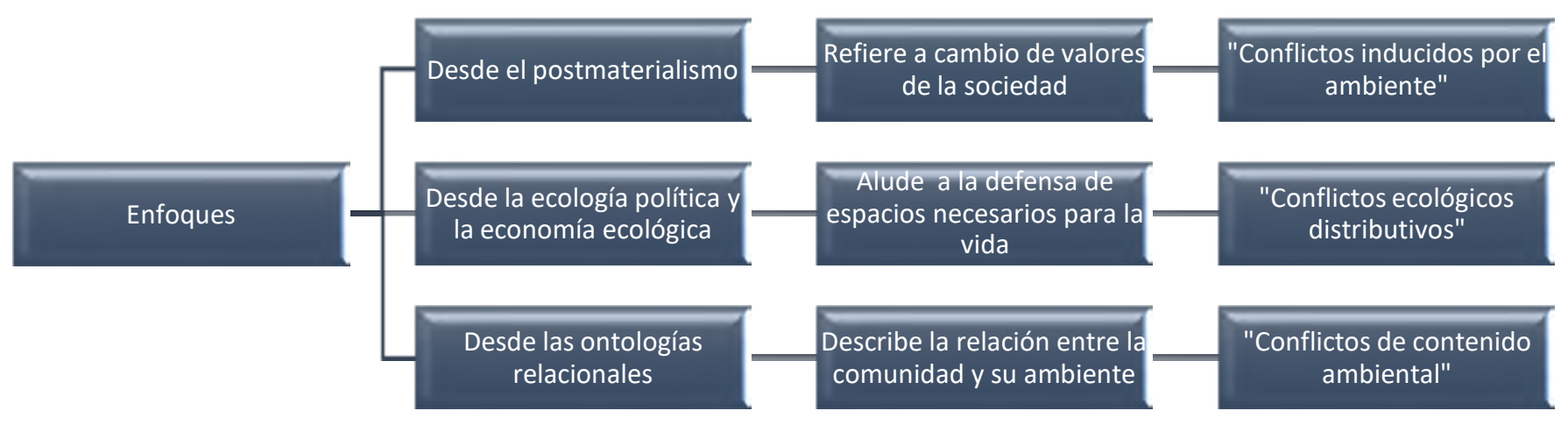

Figura 1: Enfoques sobre los conflictos socioambientales

Fuente: Adaptado de Walter (2009).

La interpretación de la figura 1 lleva a reconocer los diversos abordajes de los conflictos socioambientales que se muestran en la figura 2. Estos abordajes pueden estar orientados a las personas (con sus cosmovisiones y valores, sus necesidades y derechos), orientados a la naturaleza (que permite reconocer los derechos de la naturaleza y la ética ecológica) y los orientados al territorio (ecologismo de los pobres o ecologismo de los medios de vida) que es una categoría incluyente de las dos anteriores (que refiere a la Pachamama como expresión local de unidad humano-naturaleza y el concepto de GAIA) (Bebbington y Humphreys, 2009) Consecuentemente los conflictos socioambientales también pueden abordarse desde perspectivas antropocéntricas, biocéntricas o ecocéntricas. 


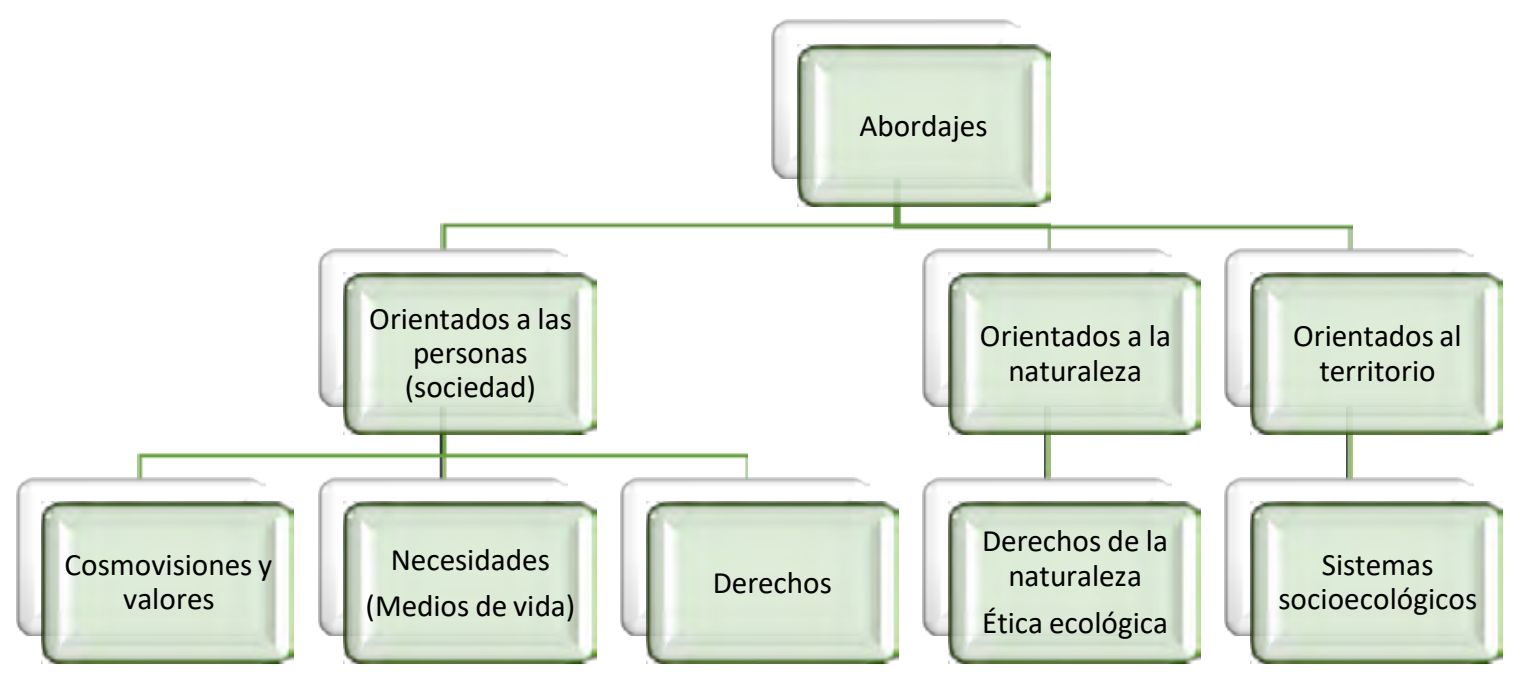

Figura 2: Diversidad de abordajes de los conflictos socioambientales

Fuente: Adaptado de Walter (2009) y Bebbington y Humphreys (2009)

Como se ha podido apreciar el enfoque de los conflictos socioambientales están fundamentalmente orientados al "uso de los recursos naturales que ofrece el ambiente" por lo tanto se inscriben en una perspectiva que Maldonado (2016a) ha dado en llamar sistemas sociales humanos. Pero en tanto estos conflictos aluden además a la naturaleza (en el sentido extenso pues incluye el cosmos) entonces también se tendría que tomar en cuenta a los sistemas sociales naturales con la salvedad que el ser humano también forma parte de estos sistemas sociales naturales. En sentido estricto la naturaleza humana es una ilusión según Sahlins (2011).

Desde una perspectiva ontológica occidental el ser humano se relaciona con una naturaleza exteriorizada y que además está al servicio de la satisfacción de sus necesidades y que se plasma en crecimiento económico o incluso en términos de desarrollo sostenible. Esta visión hegemónica invisibiliza y devalúa la naturaleza que cumple un mero fin instrumental. No obstante, cada vez se reconoce con más contundencia que no existen ni la naturaleza ni cultura como categorías aisladas sino que forman realidades entrelazadas aunque en ocasiones hay más manifestaciones de lo humano y en otras más expresiones de la naturaleza (Maldonado, 2019). Muchos atributos que eran considerados exclusivamente humanos no lo son tanto y que existen en otros seres vivos aunque, claro está con diferencias de grados. Se ha reportado por ejemplo que el moho de limo Physarum polycephalum guarda recuerdos, a pesar de no tener sistema nervioso (Romero, 2021). La neurobiología vegetal ha dado cuenta que las plantas tienen capacidad de interpretar el entorno y por tanto ajustarse a las presiones por lo que se puede reconocer inteligencia (Maldonado, 2016). Desde una perspectiva más controversial para la ciencia hegemónica Sheldrake (2021) afirma que la conciencia no es solo propiedad de los seres vivos sino que incluso se encontraría en las diversas manifestaciones del cosmos.

Una cosa es establecer relaciones con una naturaleza inerte que está al servicio de las necesidades de los seres humanos, visión que surge desde el sistema capitalista neoliberal, y otra cosa es establecer relaciones con una naturaleza que alberga vida, en todas sus expresiones, más allá de la utilidad que puedan tener para los seres humanos de la actualidad y del futuro como reza el concepto de desarrollo sostenible. La naturaleza convertida en recursos aparece entonces como insumo para el crecimiento económico y desarrollo asumiendo que es posible crecer infinitamente en un mundo de biocapacidad finita. La conciencia de la crisis global ha llevado a que se incorporen las consideraciones sociales y ambientales cada vez con mayor énfasis pero sin mover el modelo de vida, de producción y de consumo. Es revelador que la propia institucionalidad internacional señale que "la primera condición básica para una transformación que busque el bienestar y supere el desenfreno capitalista implica considerar la dimensión ambiental como sujeto político de excelencia" (Giglo, 2020, 49). Desde esta perspectiva surge la necesidad de explorar alternativas al desarrollo tales como el buen vivir o el biodesarrollo focalizado en el respeto a todas las manifestaciones de la vida (Maldonado, 2012, 2016b, 2018, 2019). 
Poner el respeto a la vida en todas sus manifestaciones cambia la lógica convencional del abordaje antropocéntrico de los conflictos socioambientales. La vida y los sistemas vivos constituyen el más complejo de los fenómenos y sistemas (Maldonado, 2019) y por ello resulta tan difícil de definirla y apenas es posible concebirla (Maldonado, 2019). Como señala Maldonado (2021, p. 200) "la vida no es una propiedad material del universo, sino una forma de organización de la materia, de la energía y de la información, una cualidad de la propia materia, energía e información que, sin embargo, no se deja reducir a cualquier de las tres expresiones físicas." Maldonado (2016, 2019, 2020) menciona que no existe ninguna diferencia ontológica o material entre la vida y no vida, entre los factores bióticos y abióticos y lo que existen son diferencias de grados cualitativos y de organización. A la fecha la vida no humana ha sido negada en el abordaje convencional del manejo de conflictos.

Una perspectiva biocéntrica apela a la ética ambiental y ecológica que amplía la comunidad moral y que es otra forma de que el ser humano se reencuentre consigo mismo como integrante de la naturaleza. Si esto es así, entonces habría que revisar el enfoque tradicional antropocéntrico de los conflictos socioambientales que solo alude a las interacciones entre humanos que se concentra en aspectos de acceso, control, uso y distribución de beneficios de los llamados recursos naturales.

Es preciso reconocer que el ser humano ha llegado a la situación actual a partir del uso de la flora y fauna silvestre, el dominio de la naturaleza a través de la domesticación de plantas y animales, la transformación de los territorios. No obstante, el hecho que las pandemias causadas por virus sean de origen zoonótico dan cuenta que la relación entre el ser humano y la naturaleza necesita ser revisada. A ello se suma el hecho que gradualmente la humanidad ha ido ganando conciencia de la necesidad de expansión de la comunidad moral, proceso aún emergente. La humanidad ha girado en torno a diversos centros entre los que se menciona la teología, el Estado, los actores privados (el mercado) y el resultado es una crisis civilizatoria (Maldonado, 2020). De ahí la importancia de explorar otras opciones en los que el eje del pensar, sentir y accionar humano se traduzca en un respeto y celebración de la vida en todas sus manifestaciones como propone el Biodesarrollo (Maldonado, 2012, 2021). Ello por supuesto sin desconocer que esta situación lleva a dilemas morales porque afectan la cultura, la alimentación, la economía.

El Convenio 169 de la Organización Internacional del Trabajo (OIT, 2007, p. 30) habla de derechos territoriales, derecho a la consulta, derecho de participación, siempre que sea posible, en los beneficios que repor- ten la explotación de los recursos existentes en sus territorios. Asimismo, tienen derecho a recibir una indemnización equitativa por cualquier daño que puedan sufrir como resultado de esas actividades (Art. 15). Una cuidadosa interpretación de estos derechos da cuenta que hay una mezcla entre los derechos indígenas que toman en cuenta su cultura y la relación de continuidad entre los pueblos indígenas y la naturaleza y una perspectiva antropocéntrica que concibe a la naturaleza como fuente de recursos para la satisfacción de las necesidades de los pueblos indígenas. Y si se dan condiciones de explotación o aprovechamiento de recursos en sus territorios tienen derecho a beneficiarse de estas actividades económicas. Estos traslapes ontológicos se pueden reducir a una transacción instrumental en la que por beneficios económicos se diluya la estrecha relación entre los pueblos indígenas y la naturaleza como efectivamente se ha podido apreciar. De esta manera se pueden reconocer conflictos de resistencia (cuando hay oposición comunitaria a la intervención de la actividad económica) y conflictos de convivencia (cuando la lucha fundamentalmente refiere a un mejoramiento de las condiciones de beneficio) (de Echave y Díaz, 2013; Tetreault et al., 2019).

Los conflictos de resistencia fundamentalmente se basan en aspectos valorativos es decir las cosmovisiones, las creencias, los significados y los sentidos, mientras que los conflictos de convivencia se asientan más en el plano material. Los conflictos de resistencia se inscriben generalmente en el marco de una ontología de continuidad a través del cual hay una relación más estrecha entre el ser humano y la naturaleza, y los conflictos de convivencia se basan en una ontología disyuntiva en la que la naturaleza es exterior y distante y cumple el rol de canasta de recursos. Es importante reconocer que estas categorías no se dan en compartimentos separados porque también se verifican entrecruzamientos. Así dentro de cada comunidad, familia e incluso persona puede haber fracturas y tensiones internas frente a una situación conflictiva. Las diversidad de posiciones frente al conflicto también pueden variar en el espacio y en el tiempo. Esta realidad da cuenta de la complejidad de los conflictos que se resiste a los encasillamientos institucionales. La misma dinámica puede verificarse en el cambio de roles entre actores primarios, secundarios y terceros que según el devenir del conflicto lo que da cuenta el carácter no lineal del proceso (Rodríguez et al., 2015).

En los conflictos socioambientales de convivencia, como se ha señalado, las pugnas y tensiones se deben a exigencias de compensaciones y una mejor distribución de los recursos económicos derivados de la actividad económica y cómo se mitigan los impactos negativos 
ambientales y sociales. Estas relaciones transaccionales se explican por la mercantilización de la naturaleza.

La fuerza ideológica y discursiva de la naturaleza como proveedora de bienes y servicios para el ser humano responde a un proceso histórico en la que el origen de la riqueza ha sido explicado desde diferentes fuentes (Polanyi, 2007; Naredo, 2017, Gómez, 2017; Castro, 2020). Un primer hito refiere a considerar la tierra (naturaleza) como origen de la naturaleza, en un segundo hito irrumpe el trabajo como factor de riqueza, en un tercer hito se legitima al capital como única fuente de riqueza y además se considera que los capitales son sustituibles. En este marco se perdió, o se vio seriamente afectada, la relación de continuidad con la naturaleza que se reduce a recursos naturales, capital natural, bienes o servicios apropiables e intercambiables en los mercados. En este proceso se originó la metáfora o ficción de la producción en la que el ser humano se apropia del trabajo de la naturaleza y la hace aparecer como creación humana y por tanto con legitimidad para incorporarla al mercado. Este es un proceso en el que convergen tanto la ciencia, la economía y la política. Es en este marco que se entiende que en los conflictos de convivencia la discusión de fondo no es sobre el extractivismo y los impactos sobre la naturaleza sino fundamentalmente cómo se consiguen mayores beneficios con los menores impactos ambientales negativos (Rodríguez et al., 2019). Este último aspecto es relativo porque hay poblados en la Sierra Central del Perú que subordinan los impactos ambientales y los aspectos de la salud de las personas a los intereses de contar con fuentes de trabajo. Desde los actores externos se escuchan voces que celebran la minería ilegal porque dan cuenta del emprendimiento de los pobres por crearse sus fuentes de trabajo (aunque invisibilicen los impactos sociales y ambientales). En un país con desigualdades y que acusa grandes bolsones de poblaciones en pobreza y extrema pobreza es posible encontrar estas contradicciones.

En el manejo de conflictos se apela a tres factores básicos para su entendimiento: Las personas, el problema y los procesos. Aunque se analizan las percepciones, los intereses, la necesidades y las acciones que realizan cada uno de los actores no se aprecia en su real dimensión la preocupación por la naturaleza y sus valores intrínsecos. Aunque en el discurso existe preocupación por la calidad de vida (o bienestar) de las comunidades el enfoque antropocéntrico domina la interpretación del conflicto. Aquí es cuando aparecen términos como "minería sostenible" (contradictorio por definición) o incluso "desarrollo sostenible" pero sin cuestionar las bases mismas del desarrollo que mantiene su apuesta por un crecimiento infinito en un planeta caracterizado por su base biofísica.

Bajo esta perspectiva manejar un conflicto es recuperar la estabilidad previamente existente, es llevarlos al campo del orden, de lo instituido (Estado de Derecho). El éxito del manejo del conflicto se basa en la capacidad de llevar a la población al campo compartido en función del modelo de desarrollo predominante. De ahí que no es raro que muchos conflictos rebroten a pesar de haber llegado a un acuerdo compartido.

\section{La complejidad de los conflictos socioam- bientales}

El hecho de hablar de conflictos socioambientales inmediatamente alude a la complejidad de los sistemas socioecológicos porque refiere a las interacciones entre los humanos, y entre los humanos y la naturaleza desde un punto de vista multi e interdimensional, multiescalar y multitemporal en el que se cruzan las diversas perspectivas, cosmovisiones, concepciones, sentires y discursos en torno a un territorio (Thomas, 2018; Morales et al., 2019). Aunque los conflictos socioambientales pueden tener una expresión local hay una estrecha conexión con las diversas escalas espaciales incluyendo la escala planetaria. Consecuentemente el abordaje de los conflictos socioambientales requiere la comprensión actual e histórica del mundo.

En los conflictos socioambientales los impactos sociales (despojo, desposesión, sacrificio de comunidades y cuerpos) y los impactos ambientales (sacrificio de la naturaleza no humana) se entrecruzan (contaminación, cambio climático, pérdida o exterminio de biodiversidad, afectación de territorios) (Van Teijlingen y Dupuits (2021). Además el conflicto socioambiental no se reduce a las pugnas por el acceso, uso y control de los llamados recursos naturales sino que se entrecruzan historias, narrativas, significados, sentidos.

Además de elementos, estructuras, se verifican relaciones no lineales que dan cuenta de una serie de fenómenos como irrupciones, crisis, quiebres, fracturas, bifurcaciones, borrosidades, incertidumbres, aleatoriedades, entre otros atributos propios del lenguaje de las ciencias de la complejidad. Los conflictos aluden a un sistema abierto no lineal en la que se verifican elementos tangibles e intangibles que se encuentran en una dinámica alejadas del equilibrio pues las interrelaciones generan vectores de encuentro, de desencuentro, de recursividad, procesos de autoorganización y propiedades emergentes. 
Ahora bien es necesario reconocer que ninguno de los actores sociales involucrados en los conflictos (primarios, secundarios y terceros) muestra una uniformidad y que existen variantes al interior de los propios actores. Aunque los Estados nacionales tienen un marco común contenido en las constituciones políticas las diversas entidades estatales también tienen sus propias orientaciones en función a sus mandatos, unas están dedicadas a la promoción del crecimiento económico, otras se dedican a la conservación de la naturaleza y su aprovechamiento sostenible mientras que otras se dedican a la defensa de los derechos de los pueblos indígenas. A ello se suman las dificultades de coordinación por los enfoques fuertemente sectoriales y a los mandatos expresos que se deben ajustar a funciones y competencias específicas. Lo mismo pasa en las comunidades por diferencias de género, de edad, de experiencias y expectativas.

\section{Discusiones}

La Conflictología es la ciencia que estudia el conflicto y comprende los conceptos de crisis, cambio, violencia o problema (Vinyamata, 2015). A diferencia de la ciencia normal que se caracteriza por ser dualista, reduccionista, mecanicista y determinista la Conflictología puede inscribirse en los enfoques de la complejidad en tanto reconoce la necesidad de superar el pensamiento dicotómico, los enfoques disciplinarios de abordaje de los conflictos, las relaciones causales, las "verdades" hegemónicas, la búsqueda de múltiples soluciones, entre otras características (Vinyamata, 2015).

No obstante es muy frecuente que el abordaje de los conflictos se haga desde perspectivas sectoriales, a lo que se suman los enfoques disciplinarios o a lo máximo enfoques multidisciplinarios como agregados de campos comunes del conocimiento, que no logran captar la complejidad de la realidad sacudida por un conflicto. El marco político institucional peruano no escapa del paradigma de desarrollo que se fundamenta en la separación del ser humano de la naturaleza y se legitima su dominio y explotación para la satisfacción de las necesidades humanas que contribuyan al crecimiento económico y el desarrollo llamado sostenible. Por lo tanto el abordaje del conflicto se hace desde los parámetros políticos institucionales que tienen como trasfondo un modelo de desarrollo capitalista neoliberal. Esta postura colisiona con un país que se caracteriza precisamente por su alta sociodiversidad con múltiples cosmovisiones, racionalidades, sentires y decires respecto a las relaciones entre la sociedad y la naturaleza (Morales et al., 2019).

En esta perspectiva el abordaje oficial de los conflictos se realiza desde una orientación antropocéntrica en el que la naturaleza queda invisibilizada como un tercer actor marginal (tercero excluido). Aunque teóricamente se toma en cuenta la diversidad cultural, e incluso se apelen a documentos publicados en idiomas de los pueblos originales, o se incluyan a intérpretes indígenas bilingües, en la práctica priman los discursos de poder con lo que se comete una serie de injusticias ambientales, sociales, culturales, epistemológicas y lingüísticas.

En los conflictos socioambientales hay que diferenciar dos situaciones: el primero referido a las personas involucradas en el conflicto y el segundo relacionado a las personas que estudian y gestionan los conflictos. Con relación a las personas involucradas en el conflicto es menester mencionar que involucra a la persona y a los colectivos en toda su identidad como entes bio-psicosociales que se ubican en un medio y que actúan. Por lo tanto no es únicamente una dimensión racional y resulta de central importancia el rol que juegan las emociones (Poma y Gravante, 2018). Quiere decir entonces que se verifica una relación territorial en el que tanto humanos y no humanos dan cuenta de procesos de dialogicidad y recursividad en los que mutuamente se influencian independientemente si se inscriban en una ontología disyuntiva o de continuidad. En la segunda situación, referida a los que estudian o gestionan los conflictos no es posible separar los sujetos externos de la realidad observada porque hay una implicación en el proceso que termina afectándolo. El actor externo se acerca a un conflicto ambiental desde su propio marco paradigmático, institucional, cultural, epistemológico, ontológico y aunque busque ser objetivo, como pretensión de la ciencia normal, su subjetividad se hace presente. El análisis de percepciones, posiciones, intereses, necesidades y actuación de los actores se hace desde sus particulares marcos.

Sea desde el Estado, los actores económicos o incluso los estudiosos de los conflictos socioambientales que comparten la ontología disyuntiva entre el ser humano y la naturaleza y la reducen a proveedoras de bienes o materias primas, o servicios en función al interés humano, una forma de mitigar los conflictos socioambientales se da a través de los programas de relacionamiento comunitario y de responsabilidad social. Pero estos enfoques, de corte más transaccional y en contextos de asimetría de poder, no logran dar cuenta de dimensiones espirituales, culturales y simbólicas que están presentes por lo que resultan insuficientes (Cohen, 2015). Aun las soluciones que se fundamentan en el diálogo no necesariamente recogen estas dimensiones que pueden ser tangibles o inmateriales. Como afirma Silva (2017 en ocasiones el diálogo se usa más bien para desconflictuar sin resolver el conflicto. 
Desde una perspectiva de gestión existe la necesidad de resolver los conflictos en el marco de la institucionalidad y el Estado de derecho que ya está configurado en un paradigma que legaliza y legitima la concepción de dominio del ser humano sobre la naturaleza en el marco del crecimiento económico y el desarrollo sostenible. Este abordaje simplificante de la realidad no permite conocer y abordar el conflicto desde su caracterización como fenómeno complejo, al que se suman la propia complejidad humana, de los ecosistemas y de las interrelaciones socioecológicas.

Incorporar el enfoque de la complejidad no significa evadir el hecho que los conflictos socioambientales deban tener respuestas coyunturales sin reducirlos a gestión de crisis. Es darle temporalidad para entender las razones históricas que han llevado a la situación conflictiva, el entendimiento de la dinámica no lineal que se verifica en la actualidad y proyectarse en las múltiples posibilidades para favorecer relaciones más armoniosas entre los seres humanos y entre los seres humanos y la naturaleza reconociendo además que forman parte de una misma realidad imbricada. En tal sentido es incorporar el devenir para fortalecer la equidad generacional e intergeneracional en perspectiva de sostenibilidad o incluso apelando las propuestas que proceden del posdesarrollo y las alternativas al desarrollo. En esta perspectiva tiene cabida los enfoques preventivos que involucren la construcción de plataformas de diálogo y de paz. Sin desconocer que los conflictos son consustanciales a las relaciones en la sociedad humana y que contribuyen a la producción de institucionalidad, entendida en sus dimensiones de edificios, normas y acuerdos. En tal sentido las consideraciones que brindan los sistemas caórdicos permiten moverse entre el orden del Estado de derecho y el caos que refiere a procesos ordenados pero altamente impredecibles. Otra versión refiere a los sistemas que se mueven entre lo instituido y lo instituyente.

Es quebrar la visión de conflictos que se reducen a desconflictuar para que el modelo económico encuentre los cauces para que las actividades extractivas e industriales sigan operando con complacencia de las comunidades afectadas por las relaciones transaccionales desarrolladas sin cuestionar la actividad económica misma. El enfoque de la complejidad de los conflictos socioambientales constituye una oportunidad para repensar los modelos civilizatorios, políticos, económicos, sociales y culturales que lleven a una relación más armoniosa y respetuosa entre los seres humanos y los seres humanos y la naturaleza (socionaturaleza). En todo este proceso está presente el tema de equidad de género que lamentablemente es un déficit en la sociedad latinoamericana.

\section{Conclusiones}

La comprensión de las relaciones entre los conflictos socioambientales y la complejidad, desde la perspectiva de las ciencias de la complejidad, ha permitido poner en evidencia el enriquecimiento del abordaje de los conflictos al ampliar significativamente los elementos, relaciones y procesos en sistemas abiertos que intercambian materiales y energía con el entorno. Entender los conflictos socioambientales en perspectiva de complejidad implica incorporar el respeto por los valores intrínsecos de la vida en todas sus manifestaciones que había sido negada o subestimada por la primacía de consideraciones de crecimiento económico y una estructura de gestión pública sectorial y disciplinaria que no da cuenta de la complejidad de la realidad. Consecuentemente surge la necesidad de revisar el modelo capitalista neoliberal hegemónico que imprime una lógica y sentido al manejo de conflictos para desconflictuar pero no necesariamente para sembrar relaciones armoniosas entre el ser humano y la naturaleza, siendo ambos expresiones de una misma realidad. Es entonces que cobra sentido la propuesta de biodesarrollo que está acompañada de una ética ambiental y ecológica.

\section{Financiamiento}

La elaboración de esta investigación no ha recibido ninguna financiación.

\section{Referencias}

Arce, R. (18 de diciembre, 2016). Transformación de conflictos en perspectiva de complejidad. SERVINDI. Transformación de conflictos en perspectiva de complejidad. Servindi - Servicios de Comunicación Intercultural

Arce, R. (4 de junio, 2018). El abordaje de conflictos sociales desde la perspectiva de la complejidad. Noticias de la Universidad Antonio Ruiz de Montoya. [Artículo] El abordaje de conflictos sociales desde la perspectiva de la complejidad (uarm.edu.pe)

Carpio, C y Meneses, S. (2006). Guía para el Manejo de Conflictos especialmente Socio Ambientales. Centro Ecuatoriano de Derecho Ambiental. LIBRO PDF (fuhem.es)

Castro, S. (2013) Pobreza, minería y conflictos socioambientales en el Perú. INTE - PUCP (Cuadernos de investigación Kawsaypacha, 1). Castro_conflictos_socioambientales.pdf (pucp.edu.pe)

Castro, A. (2018). El desafío de un pensar diferente : pensamiento, sociedad y naturaleza CLACSO.

Comissão Pastoral da Terra - CPT. (2020). Atlas de Conflictos Socioterritoriales Pan-Amazónico. CPT. ES_Atlas_de_Conflictos_Socioterritoriales_Pan-Amazonico1.pdf (forosocialpanamazonico.com) 
Cohen, M. A. (2015). Conflictos socio-ambientales: La minería en wirikuta y cananea. El Cotidiano, 30(191),97108. http://aulavirtual.urp.edu.pe/bdacademicas/scholarly-journals/conflictos-socio-ambientales-la-minería-en/ docview/1684297312/se-2 ?accountid =45097

Comisión Económica para América Latina y el Caribe (CEPAL). (2021). Construir un futuro mejor: acciones para fortalecer la Agenda 2030 para el Desarrollo Sostenible. CEPAL. Construir un futuro mejor: acciones para fortalecer la Agenda 2030 para el Desarrollo Sostenible (cepal.org)

De Echave, J. y Diez, A. (2013). Más allá de Conga. RedGE, CooperAcción. 00164.pdf (cooperaccion.org.pe)

Defensoría del Pueblo. (2021). Reporte de conflictos sociales Nro. 204. Adjuntía para la Prevención de Conflictos Sociales y la Gobernabilidad. RCS N 126 (defensoria.gob.pe).

Gligo, N, y otros. (2020). La tragedia ambiental de América Latina y el Caribe, Libros de la CEPAL, No 161 (LC/PUB.2020/11-P). Comisión Económica para América Latina y el Caribe (CEPAL). ResearchGate

Gómez Baggethun, Erik (4 de enero, 2017). Controversias y desafíos en torno a la valoración de los servicios de los ecosistemas, Norwegian University of Life Scences. IV Encuentro Internacional de Economía Ecológica. https://youtu. be/GWCUZ-hhwM4

Gómez, M. (14 de febrero de 2021). Conflictos socioambientales y conflictividades comunicacionales. Iberoamérica social. Conflictos socioambientales y conflictividades comunicacionales | Marx José Gómez Liendo (iberoamericasocial.com)

Lara, F. (2016). Las ciencias de la complejidad en la solución de nuestros problemas sociales. Sistemas, Cibernética e Informática. 13 (2), 43-50. CB076IQ16.pdf (iiisci.org)

Maldonado, C.E. (2012). Bioeconomía y biodesarrollo. El biodesarrollo: saber qué se quiere y qué necesitamos como búsqueda de un modelo alternativo. Le Monde diplomatique| el Dipló 116, 32-33

Maldonado, C. (2016a). Complejidad de las ciencias sociales. Y de otras ciencias y disciplinas. Ediciones desde abajo. (PDF) Complejidad de las ciencias sociales. $Y$ de las otras ciencias y disciplinas (researchgate.net)

Maldonado, C. (2016b). Pensar como la naturaleza una idea radical. Uni-pluri/versidad, 16(2), 41-51. https://www. researchgate.net/profile/Carlos_Maldonado2/publication/318513453_Pensar_como_la_naturaleza_Una_idea_radical/ links/59760af0aca2728d026c1e00/Pensar-como-la-naturaleza-Una-idea-radical.pdf?origin=publication_detail

Maldonado, C. (2018). Bioeconomía, biodesarrollo y civilización. Un mapa de problemas y soluciones. En M. Eschenhagen y C. Maldonado (Edit.), Epistemologías del sur para germinar alternativas al desarrollo. Debate entre Carlos Maldonado y Horacio Machado (pp: 69-93) Universidad el Rosario. Universidad Pontificia Bolivariana.. Bioeconomía, biodesarrollo y civilización.pdf (cinfopec.com.mx)

Maldonado, C. (2019). Las ciencias de la complejidad son ciencias de la vida. En M. Villegas, L. Caballero y E. Vizcaya (Eds.),
Biocomplejidad: facetas y tendencias (pp: 259- 295). CopIt-arXives, TS0018ES.pdf (unam.mx)

Maldonado, C. (2020). Occidente, la civilización que nació enferma. Ediciones desde abajo. (PDF) Occidente, la civilización que nació enferma (researchgate.net)

Maldonado, C.E. (2021). Las Ciencias de la Complejidad son Ciencias de la Vida. Primera edición. Trepen Ediciones. (PDF) Las ciencias de la complejidad son ciencias de la vida (researchgate.net)

Morales, B., Aliste, E., Neira, I. y Urquiza, A. (2019). La compleja definición del problema socioambiental: racionalidades y controversias. MAD 40 (2019): 43-51 DOI: 10.5354/07190527.2019 .54834

Naredo, José Manuel (4 de enero, 2017). Orígenes y enfoques de la economía Ecológica. Universidad Politécnica de Madrid, España, IV Encuentro Internacional de Economía Ecológica. Instituto de Estudios Ambientales. IDEA-UN. https://youtu. be/6cymP1EEruY

Organización Internacional del Trabajo. (2007). Convenio № 169 sobre pueblos indígenas y tribales en países independientes. 2a. ed. OIT, Oficina Regional para América Latina y el Caribe. Convenio 169 de la OIT.pdf (cultura.gob.pe)

Ortiz, P., Zárate, C. y Terán, J. (2011). Mirar los conflictos socioambientales. Una relectura de conceptos, métodos y contextos. Volumen I. Universidad Politécnica Salesiana. CASITA Genero y ambiente 4.qxd (ups.edu.ec)

Polanyi, Karl. (2007). La gran transformación. Critica del liberalismo económico. Quipu Editorial.

Poma, A., y Gravante, T. (2018). Emociones, identidad colectiva y estrategias en los conflictos socioambientales. Andamios, 15(36), 287-309. doi: http://dx.doi.org/10.29092/uacm. v15i36.611

Raworth, K. (2018). Economía rosquilla. 7 maneras de pensar la economía del siglo XXI. Editorial Paidós.

Rodríguez, I., Inturias, M., Volker, F., Robledo, J., y Sarti, C. y Borel, R. (2019). Conflictividad socioambiental en Latinoamérica Aportes de la transformación de conflictos socioambientales a la transformación ecológica. Friedrich-Ebert-Stiftung Proyecto Regional Transformación Social-Ecológica. ResearchGate

Rodríguez, I.; Sarti, C. y Castro, V. (Eds.). (2015). Transformación de Conflictos Socio Ambientales e Interculturalidad. Explorando las Interconexiones. Grupo Confluencias; Grupo de Trabajo sobre Asuntos Indígenas (GTAI) del Centro de Estudios Políticos y Sociales de América Latina (CEPSAL) de la Universidad de los Andes de Venezuela y Organización Regional de Pueblos Indígenas de Amazonas (ORPIA). (99+) (PDF) Transformacion de Conflictos Socio Ambientales e Interculturalidad. Explorando las interconecciones. | Iokiñe Rodriguez - Academia.edu

Romero, S. (5 de marzo de 2021). ¿Es posible tener recuerdos sin cerebro? Muy interesante. ¿Es posible tener recuerdos sin cerebro? (muyinteresante.es)

Sahlins, M. (2011). La ilusión occidental de la naturaleza humana. Fondo de Cultura Económica. 
Secretaría de Gestión Social y Diálogo Viceministerio de Gobernanza Territorial de la Presidencia del Consejo de Ministros. (2019). Reducir la conflictividad y mejorar el cumplimiento de los compromisos. Revista Willaqniki. Edición 3. W2019. cdr (pcm.gob.pe)

Sheldrake, R. (2021). Is the Sun Conscious? Journal of Consciousness Studies, 28,( 3-4), 8-28. Is_the_Sun_Conscious.pdf (sheldrake.org)

Silva, R. (2017). Mujeres y conflictos ecoterritoriales. Impactos, estrategias, resistencias. Entrepueblos, AIETI- Asociación de Investigación y Especialización sobre Temas Iberoamericanos, Demus Estudio para la Defensa de los Derechos de la Mujer, CMP Flora Tristán, Coordinadora Nacional de Derechos Humanos. (99+) (PDF) Mujeres y conflictos ecoterritoriales. Impactos, estrategias, resistencias. | Rocio Silva Santisteban Manrique - Academia.edu

Tetreault, D., McCulligh, C. y Lucio, C. (Coords.) (2019), Despojo, conflictos socioambientales y alternativas en México. Universidad Autónoma de Zacatecas. 2-Conflicto socioambiental - 2.pdf (unam.mx)
Thomas, M. (2018). Ordenamiento Territorial y Conflictos Socioambientales en el Sector Energía Análisis de la Política Energética 2050 frente a conflictos socioambientales en el Territorio de San Clemente. [Tesis presentada para obtener el grado académico de Magister en Desarrollo Urbano]. Instituto de Estudios Urbanos y Territoriales Pontificia Universidad Católica de Chile. TESIS-MITR.pdf (uc.cl)

van Teijlingen, K., y Dupuits, E. (2021). Presentación del dossier: Estrategias comunitarias frente a conflictos socioambientales: Más allá de la resistencia. Iconos, 25(1), 7-16. http:// aulavirtual.urp.edu.pe/bdacademicas/scholarly-journals/ presentación-del-dossier-estrategias-comunitarias/docview $/ 2480323184 /$ se-2 ?accountid $=45097$

Walter, M. (2009). Conflictos ambientales, socioambientales, ecológico distributivos, de contenido ambiental... Reflexionando sobre enfoques y definiciones. Boletín Ecos. 6, 1-9. untitled (fuhem.es)

WWF. 2020. Informe Planeta Vivo 2020: Revertir la curva de la pérdida de biodiversidad. Resumen. Almond, R.E.A., Grooten M. y Petersen, T. (Eds). WWF. infomeplanetavivo_2020_resumen_1.pdf (panda.org) 